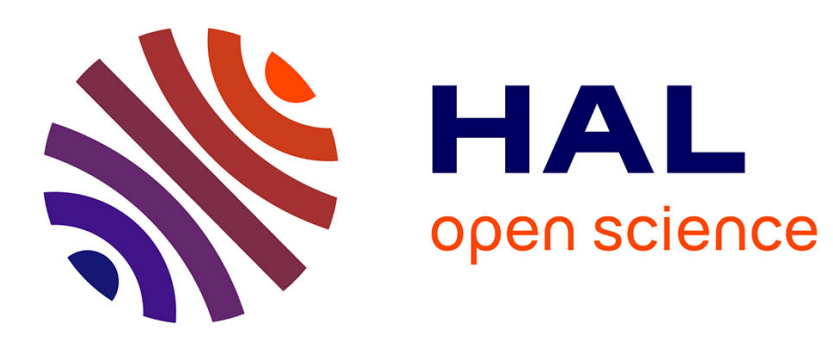

\title{
L'articulation des messages dans une publicité de parfum: Hypnotic Poison de Dior
}

Renáta Varga

\section{To cite this version:}

Renáta Varga. L'articulation des messages dans une publicité de parfum: Hypnotic Poison de Dior. Communication \& langages, 2002, 132, pp.91-103. 10.3406/colan.2002.3144 . hal-01233034

\section{HAL Id: hal-01233034 \\ https://hal.science/hal-01233034}

Submitted on 24 Nov 2015

HAL is a multi-disciplinary open access archive for the deposit and dissemination of scientific research documents, whether they are published or not. The documents may come from teaching and research institutions in France or abroad, or from public or private research centers.
L'archive ouverte pluridisciplinaire HAL, est destinée au dépôt et à la diffusion de documents scientifiques de niveau recherche, publiés ou non, émanant des établissements d'enseignement et de recherche français ou étrangers, des laboratoires publics ou privés. 


\section{L'articulation des messages dans une publicité de parfum : Hypnotic} Poison de Dior

Renata Varga

\section{Résumé}

L'article proposé par Renáta Varga constitue une étude de cas assez démonstrative de préoccupations actuelles de l'analyse sémiologique. Cela en fait un document pédagogique pour ceux qui cherchent à comprendre comment peut se construire une interprétation de document, attentive à la fois à son statut social et économique, à son matériau signifiant, aux jeux de relation entre les langages, aux différentes dimensions du sens (signification, sensation, sensibilité). Observant que le parfum présente certaines caractéristiques qui rendent paradoxale voire impossible la présentation visuelle de ses qualités (rejoignant par là le vin analysé par Yves Jeanneret et Emmanuel Souchier à partir de ses étiquettes dans le numéro 121 de Communication \& Langages), Renéta Varga décrit la sorte de transsubstantiation opérée par quelques publicités : un certain type de construction intersémiotique qui permet de rendre sensible la qualité d'un produit surtout chargé de sa volatilité (voir l'article de Patrice Tran Ba Huy, "Odorat et histoire sociale », Communication \& Langages, $n^{\circ}$ 126, 2000.

\section{Citer ce document / Cite this document :}

Varga Renata. L'articulation des messages dans une publicité de parfum : Hypnotic Poison de Dior. In: Communication et langages, n`132, 2ème trimestre 2002. pp. 91-103.

doi : 10.3406/colan.2002.3144

http://www.persee.fr/doc/colan_0336-1500_2002_num_132_1_3144

Document généré le 23/09/2015 


\title{
L'articulation des messages dans une publicité de parfum : Hypnotic Poison de Dior
}

\author{
Renáta Varga
}

L'article proposé par Renáta Varga constitue une étude de cas assez démonstrative de préoccupations actuelles de l'analyse sémiologique. Cela en fait un document pédagogique pour ceux qui cherchent à comprendre comment peut se construire une interprétation de document, attentive à la fois à son statut social et économique, à son matériau signifiant, aux jeux de relation entre les langages, aux différentes dimensions du sens (signification, sensation, sensibilité). Observant que le parfum présente certaines caractéristiques qui rendent para- doxale voire impossible la présentation visuelle de ses qualités (rejoignant par là le vin analysé par Yves Jeanneret et Emmanuël Souchier à partir de ses étiquettes dans le numéro 121 de Communication \& Langages), Renáta Varga décrit la sorte de transsubstantiation opérée par quelques publicités: un certain type de construction intersémiotique qui permet de rendre sensible la qualité d'un produit surtout chargé de sa volatilité (voir l'article de Patrice Tran Ba Huy, «Odorat et histoire sociale », Communication \& Langages, $n^{\circ} 126,2000$.

Les publicités de parfum bénéficient d'un statut particulier dans la persuasion publicitaire. Cette particularité est due à l'objet même de ce type d'annonce qui n'est autre qu'une fragrance. En effet, comment mettre en scène une «odeur » sur du papier glacé? Même si dans une annonce l'échantillon est joint ${ }^{1}$, ce dernier ne donne qu'une impression pâle de ce que sera le même parfum sur la peau d'un consommateur/d'une consommatrice. D'ailleurs, la senteur n'est pas exactement la même selon les types de peau; elle évolue aussi dans le temps en dévoilant progressivement trois types de notes: la note de tête d'abord, la note de cœur ensuite et enfin la note de fond ${ }^{2}$. Mais la représentation d'un parfum est d'autant plus difficile qu'il

1. Les échantillons sont rares dans les publicités de parfum. Sur cent annonces de moins de trois ans, nous n'avons trouvé que trois échantillons, ce qui représente un pourcentage négligeable.

2. D'après la terminologie des parfumeurs. 
reste dans le domaine du subjectif. Aujourd'hui, il n'existe pas de méthode objective pour décrire une fragrance à part l'énumération de ses composantes ${ }^{3}$.

La perception des odeurs n'est-elle pas très différente des autres types de perceptions? L'odorat est à la fois inné et culturel. Culturel dans le sens où il est sujet d'acquisition: l'enfant doit passer par un apprentissage des types d'odeurs. Sa perception dépendra fortement de son expérience et des souvenirs associés à des odeurs précises. C'est pourquoi la mémoire olfactive est une mémoire à long terme qui mobilise des souvenirs personnels, liés à l'expérience olfactive.

Compte tenu de ces spécificités, les publicités de parfum ne peuvent être focalisées sur la fragrance elle-même, mais véhiculent un message qui la valorise. L'univers des parfums étant un univers de rêve, les publicités de parfum font partie de ce que J.-M. Floch appelle une publicité mythique ${ }^{4}$. Pour lui, l'idéologie de ce type de publicité est fondée sur une valeur ajoutée qui n'est pas inhérente au produit, mais qui est créée par la publicité, l'objectif de cette dernière étant de «décaler la vision du monde ". Les publicités de parfum, démunies de la possibilité de «faire sentir la fragrance », sont donc fondées sur une autre stratégie de persuasion et construisent leur message autour d'un univers spécifique mis en scène sur le papier. Ce message doit être à la fois simple et complexe. Simple pour être compris par un grand public et complexe pour atteindre plusieurs objectifs: attirer l'attention, susciter le désir et si possible décider à l'acte d'achat. II est donc intéressant de regarder la façon dont les messages d'une annonce publicitaire de parfum sont organisés.

\section{HYPNOTIC POISON DE DIOR}

Cette étude est fondée sur l'image publicitaire d'un parfum de Dior, Hypnotic Poison (cf. photo ci-dessous). II s'agit de la publicité d'un parfum récent, diffusée sur support papier. Cette annonce presse a accompagné le lancement de ce nouveau parfum, il n'y a donc aucune interférence avec des annonces plus anciennes du même produit. Par ailleurs, la marque est

3. Ackerman D., «L'odorat », in Le Livre des sens, Paris, Grasset, 1991, p. 15-85.

4. Floch J.-M., Sémiologie, Marketing et Communication. Sous les signes, les stratégies, Paris, PUF, 1990. 
L'articulation des messages dans une publicité de parfum 93

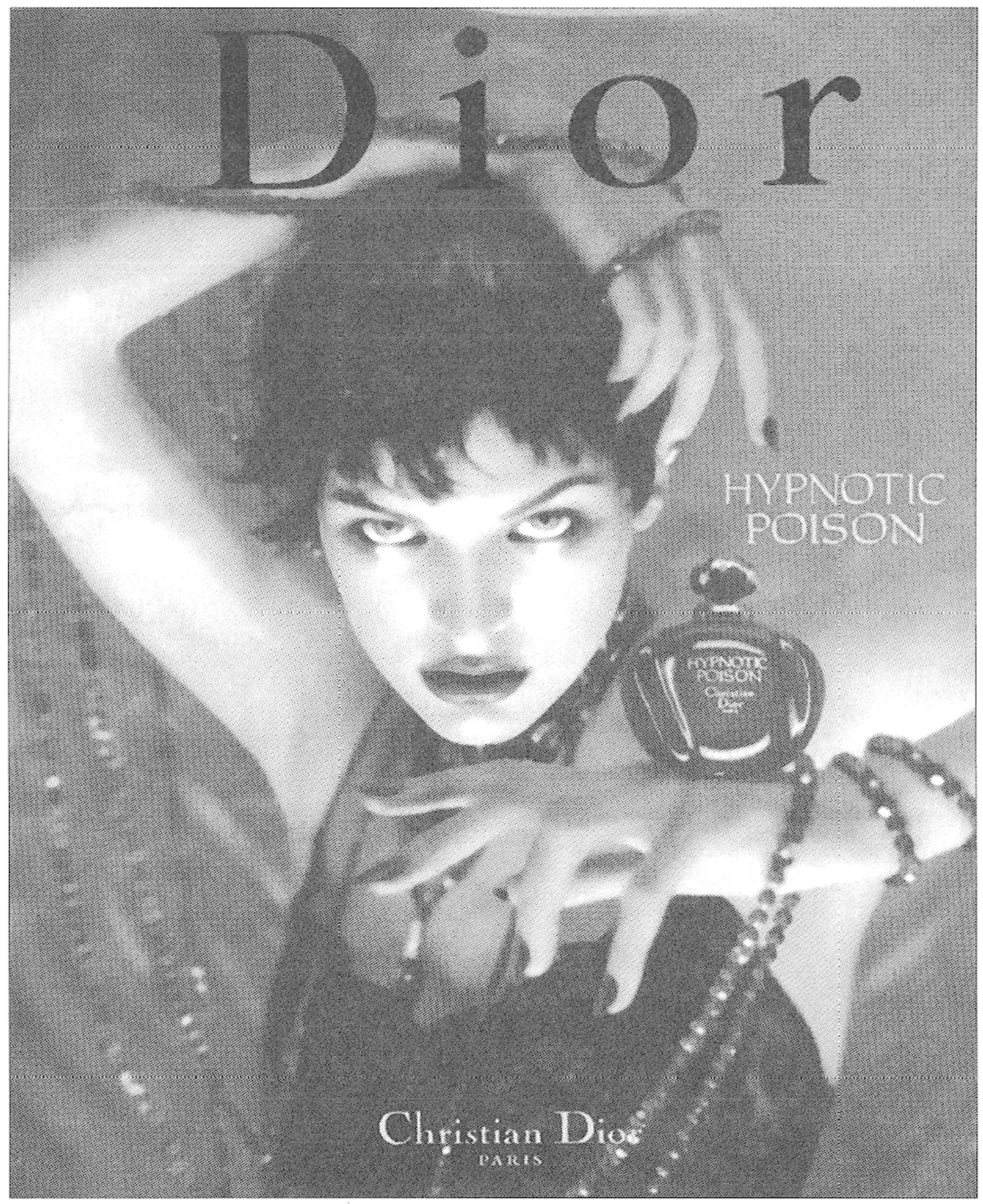




\section{Publicité}

largement connue, la perception de son image est stabilisée. L'annonce met en scène un seul parfum, ce qui facilite l'analyse de la représentation iconique de la fragrance.

À travers l'analyse de cette annonce de parfum, l'objectif est de montrer le fonctionnement du message publicitaire de ce type de produit et plus précisément, de mettre en évidence l'interaction de différents types de codes qui participent à la construction du sens.

\section{CONSTRUCTION DE L'IMAGE}

L'image de la publicité du parfum Hypnotic Poison est construite à partir de plusieurs éléments (cf. photo) :

- un personnage au centre, occupant deux tiers de l'image; sa peau est très blanche, ses yeux sont verts et il porte un maquillage rouge;

- un flacon sur la main gauche du personnage, au centre de l'image, décalé vers la droite; le corps du flacon est rouge alors que le bouchon est noir;

- un (sous-) vêtement que porte le personnage, de couleur noire;

- un collier long sur le personnage (autour du cou et sur les bras), de couleur rouge;

- un arrière-plan ou fond appelé décor, de couleur rouge;

- le nom de la marque (avec trois occurrences: une au centre en haut de l'image avec une fonction d'accroche, de couleur noire, une au centre en bas de l'image avec une fonction de « base-line », de couleur or et une sur le flacon avec une fonction d'identification), de couleur or ;

- le nom du parfum (avec deux occurrences: une sur le flacon avec la fonction d'identification et une à droite au centre de l'image avec la fonction de slogan, les deux en couleur or).

Ces éléments réunissent plusieurs types de codes qui participent à la transmission du message. D'abord le code iconique qui à travers l'image regroupe les autres types de codes. Ensuite le code linguistique, même si cette publicité, comme les annonces de parfum en général s'appuie peu sur l'écrit. L'image véhicule également le code de la marque qui porte des représentations liées à la marque Dior. Le flacon et le collier relèvent du code de l'objet, analysables à la fois à partir de leurs aspects formels (taille, forme, couleur, etc.), mais aussi sur la base des symboles qu'ils portent. Le vêtement du personnage peut être 
étudié à travers l'approche du code de l'objet, mais sa véritable symbolique est véhiculée par un code plus spécifique, celui de la mode. La représentation du personnage implique deux types de codes: le code proxémique qui est basé sur la distance du personnage par rapport au lecteur et le code kinésique dont relèvent les gestes figés par la photo. Enfin, le code olfactif est présent dans l'univers mis en scène mais n'apparaît que de façon implicite sur l'image.

Un premier aperçu rapide révèle des détails intéressants sur l'image. La technique de prise de vue est une technique de photographie, ce qui rend l'image naturelle. Néanmoins, la photo a été prise dans un studio, avec une pose du personnage très recherchée, l'univers représenté par la photo ne renvoie donc pas à la réalité.

Les points forts, les lignes de force se situent à la tête (au centre) et aux deux bras du personnage. Ces lignes sont davantage perceptibles grâce à une luminosité intense. Ces éléments attirent donc en premier le regard du récepteur et le focalisent.

L'emplacement du personnage au centre de l'image rend la publicité atemporelle. Le personnage n'est tourné ni vers la gauche, ce qui pourrait être interprété comme le passé, ni vers la droite, ce qui pourrait être perçu comme le futur. II est ancré dans le présent, mais un présent atemporel. En revanche, le flacon est légèrement décalé vers la droite, ce qui peut favoriser la perception d'un produit du futur.

Le point de fuite qui donne du relief à l'image est une ligne imaginaire qui relie le bras gauche et le bras droit du personnage via le flacon, ce qui crée un effet de profondeur au premier plan de l'image. Ainsi, le flacon semble même sortir du premier plan et se rapprocher du lecteur comme si le personnage lui proposait le produit.

La perspective est de grande échelle: le personnage prend toute la place; il se trouve à une distance personnelle du lecteur. Ce dernier se retrouve donc dans la zone intime du personnage qui s'impose en fixant son regard perçant sur lui ${ }^{5}$. II

\footnotetext{
5. A. Rosenbaum remarque à propos des publicités que les personnages représentés sur les annonces cherchent très souvent à capter le regard des récepteurs et que la persuasion publicitaire devient de plus en plus un combat entre l'œil et l'image. Rosenbaum A., "Le devoir du regard", in Communication \& Langages, $n^{\circ} 117$, Paris, 1998, p. 28-34.
} 
n'y a pas d'échappatoire possible. C'est un face à face redoutable. La communication est établie entre le lecteur et le personnage dont le comportement et le regard sont chargés de sens multiples.

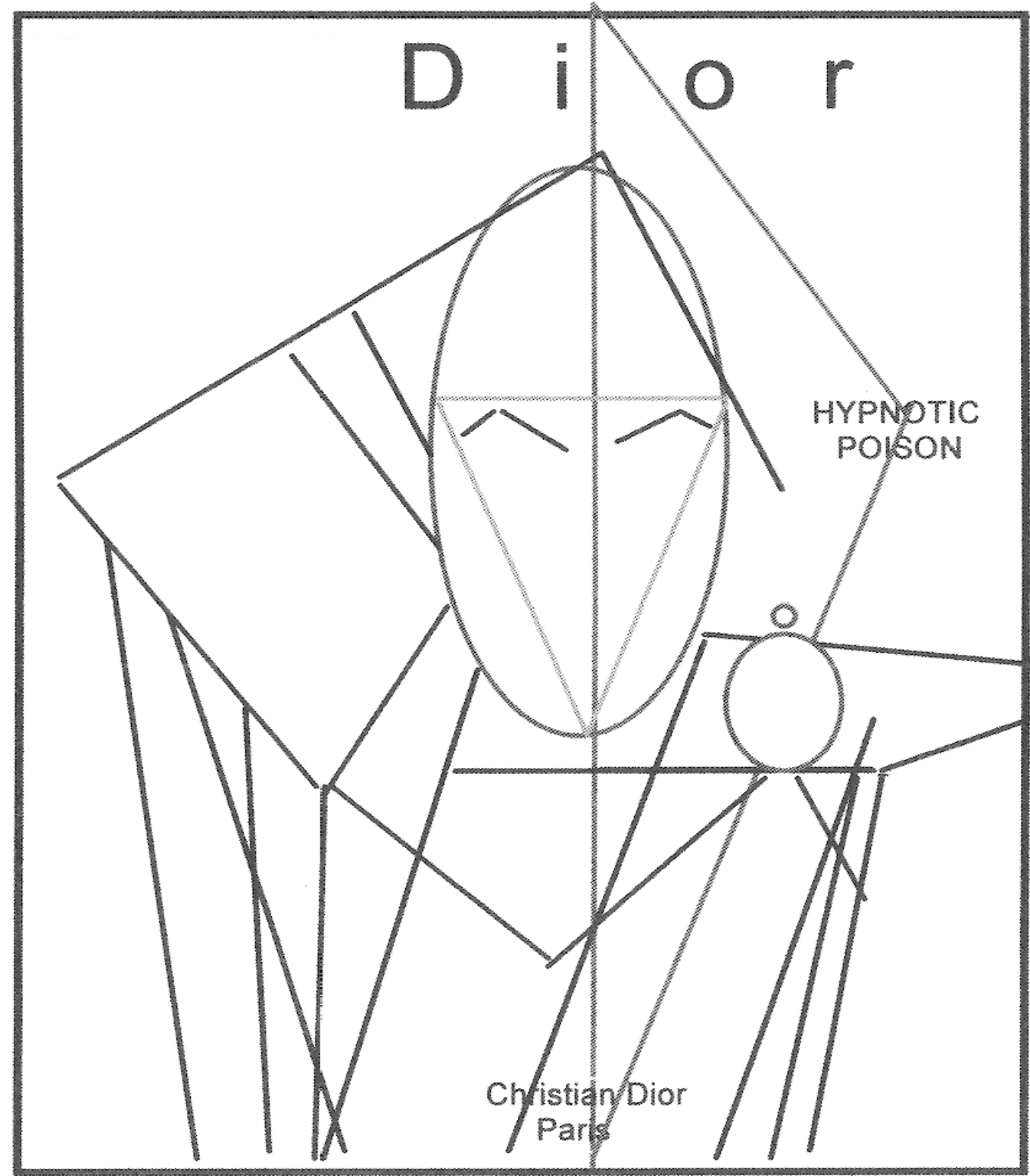

Les lignes qui parcourent l'image sont de différentes natures (cf. figure ci-dessus) et suggèrent plusieurs significations :

- les lignes obliques, très nombreuses, sont constituées par le collier, les bras et le corps du personnage et inspirent un effet de dynamisme; 
- parmi les volumes et figures géométriques: les cercles composés de la tête du personnage, de la forme du flacon, du bouchon du flacon et des lettres « $D$ » et «O $O$, de forme arrondie, renvoient à la perfection;

- le triangle imaginaire dessiné sur le visage évoque la féminité et plus précisément il est le symbole du sexe féminin; celui reliant les quatre unités textuelles, pointe à droite, renvoie au concept d'harmonie.

La perception de ces lignes et figures n'est pas forcément consciente mais guide l'interprétation du message.

\section{INTERPRÉTATION DU MESSAGE}

Le message de cette image publicitaire s'organise autour de plusieurs champs sémantiques. Dans chaque champ sémantique, on peut identifier de nombreux réseaux de signification qui sont imbriqués les uns dans les autres et qui véhiculent des messages différents. Ces réseaux de signification sont fondés sur les différents éléments de l'image au niveau dénotatif ou à des sens symboliques attachés à ces éléments. Ces réseaux véhiculent des contenus différents et participent ensemble à la construction du message.

Dans l'annonce analysée, quatre champs sémantiques peuvent être distingués: le premier s'articule autour du thème de la sorcellerie, le deuxième est lié à la féminité, le troisième est relatif au code olfactif et le quatrième s'attache au concept de luxe. Ils sont classés par ordre décroissant selon le nombre de significations véhiculées.

\section{Thème de la sorcellerie}

Le réseau qui est le noyau des messages construits autour du thème de la sorcellerie regroupe les unités, dont l'ensemble dénote un regard paralysant, hypnotisant. Les éléments iconiques qui y participent se focalisent autour des yeux et se combinent avec la mimique du personnage (expression des sourcils) et sa distance intime par rapport au récepteur. La combinaison de ces éléments mobilise les codes iconique, kinésique et proxémique. La posture de la tête (penchée légèrement vers le lecteur) est le résultat d'une prise de vue en contreplongée qui magnifie le personnage et suggère que le lecteur lui est soumis. La luminosité qui se focalise autour des yeux 
attire l'attention en premier sur ces éléments. Les yeux comme des aimants attirent et maintiennent le regard du lecteur. Le terme «hypnotique» qui renvoie à l'hypnose et le symbole de l'hypnose, mis en évidence par la posture des bras (qui ressemble à un « 8 » retourné), contribuent également à l'émergence de cette signification.

Le réseau précédent, combiné avec d'autres éléments sert d'appui à d'autres significations. Les grands yeux verts, les bras tordus et les doigts écartés du personnage, ainsi que la forme allongée de ses ongles qui dans ce contexte connotent « griffes ", renvoient à un chat prêt à griffer.

La couleur rouge du décor qui dans ce contexte connote «sang » peut également participer à ce réseau de contenus et modifie légèrement la signification précédente: il s'agit alors d'un félin prêt à attaquer.

La forme, la longueur et la disposition du collier autour du cou et du corps du personnage évoquent un serpent. Le chat prêt à attaquer peut être un chat noir, ce que suggère la couleur des cheveux et du vêtement du personnage. Cet animal porteur de symboles négatifs, l'image du serpent et le visage de femme ainsi que le concept «poison » renvoient à une sorcière.

Le concept de parfum est mis en évidence par de nombreux éléments : la publicité elle-même, le flacon, le nom du parfum et le nom de la marque. Ce concept associé à l'image d'une sorcière renvoie à la notion de magie et à l'instrument de la magie. Cette association n'est point hasardeuse : au Moyen Âge, les parfums ont été utilisés pour ensorceler ${ }^{6}$.

Si l'on regarde l'histoire de la civilisation occidentale, le parfum a toujours occupé une place importante: il a été considéré comme un lien entre la vie terrestre et l'au-delà, à la fois comme lien avec Dieu et le Diable d'où son ambiguïté et le mysticisme qui l'a toujours entouré. L'étymologie du mot rappelle ce lien: «par/fum » signifie «au-delà/des fumées ». Cette étymologie évoque l'encens brûlant dans les églises pour faciliter la communication avec l'au-delà. Cette publicité met en scène exclusivement le lien avec le Diable. D'ailleurs, le lecteur est le témoin sinon la victime d'une véritable descente en Enfer: les 
significations présentées dans le paragraphe suivant se focalisent autour du monde de l'au-delà'.

Le personnage-sorcière avec la couleur rouge et la texture du décor du fond évoquent le feu. La blancheur excessive de la peau du personnage est en contraste avec la couleur rouge de ses lèvres et la couleur noire de ses vêtements. Le jeu de lumières attire l'attention sur son visage pâle. Dans ce contexte macabre, cette blancheur associée à un «poison » renvoie à la mort. Le concept de mort et de feu avec le jeu de lumières sur le décor du fond rappellent l'enfer. La coupe de cheveux du personnage très courte avec des mèches rebelles et noires évoque le concept de diable. Celui-ci, associé au visage de jeune femme, privilégie l'interprétation d'une diablesse. Dans ce contexte, les pierres rouges du collier renvoient au rubis qui est la pierre de l'amour envoûtant ${ }^{8}$. Cette signification additionnée au concept de parfum renvoie à la magie noire.

Ces réseaux de signification se combinent entre eux et sont construits les uns sur les autres. Néanmoins, il est impossible de prédire la façon dont le récepteur décode ces messages. Même si ces contenus reposent les uns sur les autres, le parcours de l'image et l'ordre d'interprétation peuvent être différents. II n'est d'ailleurs pas certain que le récepteur perçoive de manière consciente la totalité des significations mises en avant par cette analyse.

\section{Thème de la féminité}

Parmi les messages relevant du champ de la sorcellerie, un certain nombre incluent l'image d'une femme: une sorcière, une diablesse; le chat et le félin peuvent traditionnellement avoir une représentation de femme. Ces représentations féminines sont toutes négatives car elles inspirent l'idée de la sauvagerie, de la violence (avec le sang) ou de la supercherie (avec le poison), de mauvaises intentions. Mais il existe en outre un certain nombre de réseaux de signification qui sont centrés exclusivement sur l'idée de la féminité.

7. Cornu G., Sémiologie de l'image dans la publicité, Paris, Les Éditions d'Organisation, 1990.

8. Chevalier J., Dictionnaire des symboles, Paris, Dunod, 1997. 
Les lignes obliques qui traversent l'image (cf. figure) renvoient au concept de dynamisme. La posture du personnage, son âge approximatif (18-20 ans) ainsi que sa coupe de cheveux véhiculent l'image de la jeunesse et de la modernité.

Les unités qui renvoient à la féminité sont nombreuses: les cercles et le triangle pointe en bas connotent le concept de féminité et les rondeurs féminines (cf. figure). Le sous-vêtement, les lèvres pulpeuses, rouges, les ongles longs et rouges, même le flacon du parfum sont associés à ce concept.

L'intimité est suggérée par les sous-vêtements du personnage et son corps tronqué sur la photo à cause de la distance personnelle entre elle et le lecteur. Les lèvres entrouvertes et rouges, pulpeuses suggèrent l'érotisme. Cet érotisme est à la fois affiché et suggéré. Affiché par le maquillage très fort et suggéré par le sous-vêtement qui ne laisse pas voir les formes de la jeune femme. Ses seins se laissent deviner par un jeu d'ombres sur sa poitrine. L'idée de l'intimité, de l'érotisme, les ongles longs et rouges, la proximité ainsi que le regard interpellant du personnage, et le concept du parfum renvoient à la séduction, à la liberté, à une femme séductrice, à une femme fatale, etc.

La forme, et la couleur du flacon ainsi que l'association liée au concept de serpent supportée par l'image du collier peuvent renvoyer à la pomme de Eve et à la légende du paradis perdu. II est important de souligner que la seule association à Dieu est en réalité un clin d'œil au Diable et que Ėve est porteuse de symboles négatifs. La jeune Ėve de la publicité semble offrir la pomme au lecteur, mais l'image est en contradiction avec l'innocence supposée de Ėve. Cette Ėve moderne est différente, c'est une empoisonneuse.

Lors de l'interprétation de l'image, les différents types de messages sont décodés ensemble et ne sont pas séparés par le lecteur. Ce dernier a une lecture globale du message. II est donc évident que lors de l'interprétation, même les significations positives comme la jeunesse, la modernité, la féminité, la séduction, etc., deviennent négatives car les connotations négatives sont plus nombreuses que les connotations positives. Ainsi, la jeune fille érotique et féminine devient un «garçon manquée", une sauvage qui fait des garçons ce qu'elle veut. La publicité véhicule donc l'image d'un nouveau type de femme : très femme, très sûre d'elle et très cruelle. 


\section{Thème du parfum}

L'objectif de la publicité est de promouvoir un parfum. Néanmoins, peu d'éléments renvoient au concept de parfum et encore moins à une fragrance. Le concept du parfum est véhiculé par l'image du flacon du parfum, le nom de la marque et le nom du parfum. L'image du flacon, le nom de la marque et le nom du parfum sont les unités qui participent au réseau de signification fondé sur le code olfactif, autrement dit ils suggèrent un parfum spécifique. Mais ces éléments donnent peu d'indications sur la nature du parfum. La forme du flacon renvoie à une fragrance intense et très féminine. Seule la couleur rouge qui traverse toute l'image renvoie à un type d'odeur: une fragrance forte, épicée et sucrée. Mais ces références sont peu précises.

II faut néanmoins remarquer qu'en choisissant le nom Hypnotic Poison, la maison Dior fait une référence à une gamme de parfums classique: «Poison » et «Tendre Poison ». Le choix du nom classe ce parfum dans la gamme, il montre donc son appartenance à un concept fédérateur tout en indiquant sa différence. D'ailleurs le flacon de Hypnotic Poison est le même que celui des deux autres, seule la couleur change. Alors que les deux autres sont de couleur verte, celle-ci est rouge. Les références entre ces couleurs peuvent être multiples. On peut penser que la couleur verte peut renvoyer à un poison, la couleur rouge, elle, est complémentaire de la couleur verte. Elle peut servir à montrer que le concept reste le même bien que le parfum reste différent. Si l'on pense à un fruit, la couleur rouge renvoie à un fruit mûr, donc à une femme qui affirme sa féminité.

Néanmoins, pour percevoir ces références, il faut que le lecteur connaisse les deux autres parfums. Mais même s'il les connaît, il n'aura pas plus d'indices sur la nature de la fragrance de Hypnotic Poison dans la mesure où elle ne ressemble pas aux deux autres. Ce choix de dénomination a un objectif commercial: offrir un nouveau produit tout en indiquant qu'il a sa place parmi les grandes classiques.

\section{Thème du luxe}

Le dernier type de message est lié à la notion de luxe et de richesse. Ce réseau est assuré par plusieurs éléments. La jeune fille est habillée d'un sous-vêtement noir de bonne qualité 
qui semble être en matière noble. Ce sous-vêtement ne représente pas la mode actuelle, il représente une mode atemporelle: très élégante et qui peut se porter à toute époque. La jeune fille porte aussi un collier rouge qui est peut-être en pierre précieuse. Ce bijou est certes long, mais très fin, il rappelle la mode des années vingt-trente. Le flacon de parfum semble être un accessoire élémentaire. La simplicité des éléments représentés sur l'image ainsi que leur qualité renvoient donc à l'idée de luxe et de prestige.

Le nom de la marque réfère à la même idée. Le terme «Dior» est disposé en haut au milieu, en grands caractères noirs, en typographie simple en guise d'accroche, mais aussi en bas, en caractères plus discrets mais en couleur or, ce qui renvoie à la richesse. II faut remarquer que la maison Dior a fondé sa stratégie de communication sur cette simplicité. La typographie qu'utilise la marque est stable. Sur les publicités de parfum le nom de la marque «Dior» se trouve toujours en haut au milieu en grands caractères ; le nom et leur siège "Christian Dior Paris " sont généralement représentés en bas de la page en plus petits caractères. L'empreinte de la marque est stable d'une publicité à l'autre, seule la couleur des caractères change. Sur cette publicité, les caractères arrondis de la marque: le «c $c$, le « $d$ » et le « $O$ » renvoient au concept d'harmonie tout comme les occurrences d'unités textuelles qui forment triangle (cf. figure). Le nom du parfum apparaît en couleur or, il est dessiné dans une typographie recherchée, connotant le prestige. Les couleurs rouge, noir et or renvoient à cette même idée. II y a un mélange savant de contrastes de couleurs: noir sur rouge, rouge sur noir, or sur noir et or sur rouge. La simplicité des éléments est contrebalancée par la richesse des couleurs.

\section{CONCLUSION}

Les interprétations identifiées révèlent que les réseaux de signification se construisent de façon très complexe. II est remarquable qu'une image publicitaire construite de peu d'éléments puisse porter autant de significations. Tous les codes participent à la construction de ces réseaux de contenus. Ainsi, le message ne peut fonctionner que par l'interaction de tous les types de codes, même s'il y a une hiérarchie dans leur importance et dans leur degré d'interdépendance. 
Dans la construction du message, le code iconique reste le plus exploité, ce qui n'est pas étonnant car il s'agit d'une image publicitaire. Le code kinésique occupe la deuxième place car les gestes et les postures jouent un rôle primordial dans les significations organisées autour de la sorcellerie et de la féminité. Le code linguistique et le code de l'objet sont en troisième place, exploités essentiellement dans ces deux champs sémantiques. Enfin, le code proxémique et le code de la marque restent minoritaires.

L'analyse de cette image publicitaire montre également que la référence au parfum ne joue qu'un rôle négligeable dans la transmission du message, par rapport aux autres messages, ce qui est contraire au point de vue de $M$. Julien ${ }^{9}$ qui pense que dans une publicité de parfum, tous les codes, tous les éléments participent à la construction d'un message qui doit traduire le code olfactif et donne donc des indications plus ou moins précises sur la fragrance. En réalité, dans cette publicité, comme dans d'autres publicités de parfum, semble-t-il, l'objectif n'est pas de vendre un parfum, mais de vendre une identité. Une identité que le parfum symbolise. L'identité de Hypnotic Poison est une femme qui incarne à la fois une femme féroce (femme-animal), une femme sorcière (qui envoûte les hommes), une femme sensuelle, érotique et séductrice, une femme fatale et manipulatrice, une femme jeune et moderne, une femme élégante et chic, une femme dynamique et active. Autrement dit, il s'agit d'une femme qui est maître de soi et maître(esse) des autres, qui manipule les hommes notamment.

Les interprétations offertes par l'analyse correspondent au discours que tient l'annonceur sur ce parfum. En effet, pour Dior, cette fragrance est « un parfum diabolique et mystérieux, philtre magique des temps modernes, qui exprime la sensualité de notre époque. Extravagant et envoûtant, Hypnotic Poison est le parfum provocant de la féminité et de l'audace ${ }^{10} »$.

Renáta Varga 